\title{
Huguenots et protestants en France
}

À propos de :

CABANEL Patrick, Histoire des protestants en France, $\mathrm{XVI}^{\mathrm{e}}-\mathrm{XXI}^{\mathrm{e}}$ siècle, Paris, Fayard, coll. « Histoire », 2012, 1502 p.

FATH Sébastien, Willaime Jean-Paul (dir.), La nouvelle France protestante. Essor et recomposition au XxI ${ }^{\mathrm{e}}$ siècle, Genève, Labor et Fides, coll. « Religions et modernités », n 9, 2011, 484 p. FATH Sébastien, Les Fils de la Réforme. Idées reçues sur les protestants, Paris, Éditions Le Cavalier Bleu, 2012, 208 p.

\section{Étienne Fouilloux}

\section{(Q) OpenEdition}

\section{Journals}

\section{Édition électronique}

URL : http://journals.openedition.org/assr/25404

DOI : $10.4000 /$ assr.25404

ISSN : $1777-5825$

Éditeur

Éditions de l'EHESS

Édition imprimée

Date de publication : 30 décembre 2013

Pagination : 59-65

ISSN : 0335-5985

Référence électronique

Étienne Fouilloux, « Huguenots et protestants en France», Archives de sciences sociales des religions [En ligne], 164 | 2013, mis en ligne le 20 février 2017, consulté le 01 mai 2019. URL : http:// journals.openedition.org/assr/25404; DOI : 10.4000/assr.25404

Ce document a été généré automatiquement le 1 mai 2019.

(C) Archives de sciences sociales des religions 


\section{Huguenots et protestants en France}

À propos de :

CABANel Patrick, Histoire des protestants en France, $\mathrm{XVI}^{\mathrm{e}}$-XXI ${ }^{\mathrm{e}}$ siècle, Paris, Fayard, coll. « Histoire », 2012, 1502 p.

FATH Sébastien, WiLlaime Jean-Paul (dir.), La nouvelle France protestante. Essor et recomposition au xxi ${ }^{\mathrm{e}}$ siècle, Genève, Labor et Fides, coll. «Religions et modernités », $\mathrm{n}^{\circ}$ 9, 2011, $484 \mathrm{p}$.

FATH Sébastien, Les Fils de la Réforme. Idées reçues sur les protestants, Paris, Éditions Le Cavalier Bleu, 2012, 208 p.

\section{Étienne Fouilloux}

1 Le protestantisme paraît avoir le vent en poupe dans la France d'aujourd'hui. Après la célébration éclatée du cinquième centenaire de la naissance de Calvin en 2009, étudiée par André Encrevé dans La nouvelle France protestante, plusieurs manifestations ont illustré cette vitalité en 2013 : à Grenoble en juillet, la seconde édition du «Grand Kiff » pour les jeunes et surtout, en septembre à Paris, le second rassemblement « Protestants en fête ». Alors que se profile à l'horizon le cinquième centenaire de l'affichage par Martin Luther de ses quatre-vingt-quinze thèses sur la porte de la chapelle du château de Wittenberg en 1517, acte de naissance de la Réforme, ces manifestations ont rompu avec le manque d'exposition médiatique dont se plaint souvent une minorité peu audible dans le concert national. Elles accompagnent des recompositions internes importantes, bien que plus discrètes : rapprochement de l'Église de la Confession d'Augsbourg et de l'Église réformée dans l'Union des Églises protestantes d'Alsace et de Lorraine (2006), fondation du Conseil national des évangéliques de France (2010) qui rassemble une bonne partie de cette mouvance effervescente (Aurélien Fauches dans La nouvelle France protestante) et union, au synode de Lyon en mai 2013, de l'Église réformée de France et de l'Église évangélique luthérienne de France (de l'intérieur) pour former l'Église protestante unie de France. Ce regain de visibilité est sensible aussi dans l'historiographie, car ces diverses occasions 
étaient bonnes pour faire le point sur l'état du protestantisme hexagonal aujourd'hui, mais aussi sur son histoire.

La première tâche est revenue à un colloque organisé du 18 au 20 novembre 2010 à Paris, dans les locaux du Sénat, à l'initiative de la Fédération protestante de France, dont les actes ont été publiés par les sociologues Jean-Paul Willaime et Sébastien Fath. Appuyés sur un sondage administré au printemps 2010 par l'IFOP à un échantillon représentatif de sept cents protestants de France métropolitaine, dont les résultats sont amplement présentés et commentés en annexe par Jean-Paul Willaime, ces actes fournissent une photographie documentée du protestantisme dans l'Hexagone au début du XXI ${ }^{\mathrm{e}}$ siècle.

3 Leur premier et principal enseignement réjouira ceux des protestants de vieille souche, inquiets de la "précarité » de leurs Églises naguère pointée par Jean-Paul Willaime ( $L a$ précarité protestante, Genève, Labor et Fides, 1992), qui craignaient d'appartenir à une espèce en voie de disparition. Non seulement le protestantisme se maintient dans la France d'aujourd'hui, mais il tend à se développer, alors que le catholicisme subit un net recul. Les protestants demeurent minoritaires, mais dans un pays où la majorité catholique est à la peine. Certes les estimations chiffrées varient d'un auteur à l'autre : à l'image de Sébastien Fath, les optimistes incluent dans leur comptage la catégorie ambiguë de ceux qui, en réponse aux sondeurs, se disent sympathisants ou " proches » du protestantisme : des catholiques mal à l'aise dans leur Église, le plus souvent, mais qui ne font pas le saut d'un rattachement formel au protestantisme. Plus réalistes, Claude Dargent, Jean-Daniel Roque, et aussi Patrick Cabanel au terme de son parcours historique, s'en tiennent à ceux, pratiquants ou non, qui se réclament explicitement d'une Église de la Réforme. En revanche, Patrick Cabanel semble quelque peu laxiste dans l'attribution de la qualité protestante à des personnalités du monde de la culture qui ont fait profession d'athéisme (Jean-Paul Sartre?). Sur cette base on peut estimer qu'avec deux millions de fidèles, le protestantisme représente $3 \%$ de la population française, « du jamais vu depuis la veille de la Révocation » de l'édit de Nantes en 1685, fait justement remarquer Patrick Cabanel (p. 1176). Ombres à ce tableau flatteur ? Les Églises continuent d'entretenir des relations compliquées avec les mouvements de jeunesse qui constituent leur avenir, mais dont les membres ne se bousculent pas dans les cultes (Arnaud Baubérot). Bien que traités le plus souvent avec égard, les protestants demeurent invisibles dans la grande presse, en dehors de quelques exceptions ponctuelles et des Dernières nouvelles d'Alsace ou... du quotidien catholique La Croix (Blandine Chelini-Pont).

4 Ce dynamisme ne concerne pourtant pas tout l'Hexagone, ni toutes les Églises se réclamant de la Réforme. Les vieilles paroisses rurales continuent de dépérir alors que les paroisses urbaines se multiplient, dans les grandes villes de province et en région parisienne plus encore. Par voie de conséquence, une lente érosion des Églises luthéroréformées disséminées dans un tissu catholique en voie de sécularisation se poursuit, avec des procédures de regroupement des paroisses semblables à celles de nombreux diocèses catholiques. Cette évolution dans l'espace traduit en fait le déplacement du centre de gravité confessionnel du protestantisme en France. Le second enseignement du colloque de 2010, surtout pour quelqu'un qui n'est pas protestant de souche, est la modification du rapport des forces interne en faveur de la nébuleuse évangélique. Certes, celle-ci n'est pas une création ex nihilo, puisque son courant piétiste-orthodoxe, importé de Suisse ou des pays anglo-saxons au XIX ${ }^{e}$ siècle, puise ses racines dans les rameaux radicaux des Réformes du Xvi $\mathrm{I}^{\mathrm{e}}$ siècle : il est donc aussi «historique » que les Églises se réclamant de Luther ou de Calvin (Christopher Sainclair). Tel n'est pas le cas de la 
mouvance pentecôtiste-charismatique dont l'expansion récente prouve que la France n'échappe pas à l'un des mouvements religieux majeurs de notre temps, même s'il est plus visible aux États-Unis ou en Amérique latine (Jean-Yves Carluer). Les évangéliques représentent aujourd'hui $40 \%$ des protestants en France, contre $60 \%$ pour les luthéroréformés. Mais la proportion est largement inversée dans les temples et les salles de prière en fin de semaine, car les évangéliques font de la participation au culte une quasiobligation et un marqueur d'appartenance à la communauté, bien que le sondage ait repéré des évangéliques non pratiquants. Ceux-ci sont également très présents sur le net, où ils constituent de véritables églises virtuelles (Pierre-Yves Kirschleger). La comparaison avec un bilan effectué en 2002 montre que l'évolution de l'historiographie reflète un tel déplacement. Alors qu'on attend toujours des travaux de recherche approfondis sur des sujets aussi importants que la création de la Fédération protestante de France au début du xxe siècle ou de l'Église réformée de France, à Lyon en 1938, les thèses d'histoire et de sociologie, mais aussi d'ethnologie ou de géographie, se multiplient sur la mouvance évangélique qui interpelle les sciences humaines dans un pays trop vite jugé déchristianisé. Au fil des actes du colloque, on découvre ainsi des Églises assez éloignées des stéréotypes tenaces sur le protestant, bourgeois austère et réservé, que Sébastien Fath, principal artisan de la réévaluation historiographique de l'évangélisme en France, pourfend avec bonheur dans la réédition de ses Fils de la Réforme. Églises de convertis, populaires et précaires qui essaiment dans une ville marquée par le protestantisme comme Montpellier, où elles attirent des fidèles d'origines diverses (Frank La Barbe); églises ethniques de l'agglomération parisienne, afro-caribéennes (Frédéric Dejean) ou asiatiques, comme ces curieux protestants wenzhou, originaires d'une même ville de Chine (Pan Junliang).

5 On a beaucoup insisté sur les différences entre ces Églises de professants et les Églises de multitude plus anciennes. Un troisième enseignement du colloque de 2010 tend à relativiser ces différences au profit d'un regain de cohésion. Certes les évangéliques restent plus conservateurs que les luthéro-réformés tant en matière politique qu'en matière de morale sexuelle: refus de l'interruption volontaire de grossesse, méfiance envers l'homosexualité et envers les manipulations génétiques. Mais le débat sur le «mariage pour tous » montre que le libéralisme des seconds a sans doute été surestimé, au moment où s'estompe leur vote pour les formations politiques de gauche. Quant aux évangéliques, ils sont bien plus présents que naguère dans les relations œcuméniques, y compris entre protestants (Jean-Paul Willaime), mais surtout dans l'action sociale, où leurs réseaux travaillent en émulation avec ceux de l'Entraide protestante (Sébastien Fath), et dans l'accueil des migrants pour lequel les initiatives d'origine protestante, comme la CIMADE, font référence (Bernard Boutter). Leurs liens avec le monde évangélique à travers la planète prolongent un cosmopolitisme protestant que ses adversaires ont si souvent dénoncé. De ce point de vue, le bilan proposé par le colloque de 2010 paraît plus nuancé. Si des Églises françaises maintiennent cet héritage à l'étranger, en Angleterre par exemple (Sophie-Hélène Trigeaud), l'influence du protestantisme français est faible en Amérique latine, exception faite de la saga du théologien de la libération Georges Casalis au Nicaragua sandiniste (Jean-Pierre Bastian). La francophonie protestante s'organise, pour contester l'hégémonie anglo-saxonne (Jean-François Zorn), mais les protestants français, pourtant très favorables à l'unité de l'Europe, ont du mal à s'y faire entendre (Bérengère Massignon). 
6 Riche d'annexes précieuses (cartes et tableaux, copieuse bibliographie « indicative »), La nouvelle France protestante est appelée à devenir un instrument de référence, non seulement pour les chercheurs en sciences des religions, mais pour les responsables ecclésiaux, protestants ou pas. Tel est le sort qu'on souhaite aussi à la «brique » de Patrick Cabanel, pour peu que sa taille, et son poids, ne découragent pas les utilisateurs potentiels : plus de 1500 pages, dont 1188 de texte, 180 de notes en fin de volume, 70 de bibliographie et près de 50 d'index. Il faut savoir gré à l'éditeur de ne pas avoir été effrayé par une telle masse! Et que dire de l'auteur qui a produit cet effort colossal ? Le premier depuis Samuel Mours, qui avait mis treize ans pour venir à bout de la tâche (1959-1972), il a relevé le pari d'écrire une histoire qui engrange plusieurs décennies de recherches sur le champ bien défriché de la Réforme en France depuis le XVI ${ }^{\mathrm{e}}$ siècle. Des histoires de la partie moderne de cette saga existaient, et plus encore des histoires de sa partie contemporaine, mais pas de synthèse des deux. Avant d'oser s'attaquer à ce monument de science et d'intelligence, il faut donc saluer la prouesse accomplie : non seulement Patrick Cabanel a tout lu sur son sujet, des thèses anglo-saxonnes au moindre article dans une revue régionale, comme en témoignent ses notes, mais il a digéré cette profusion avec un bonheur d'écriture, voire une verve, qui ne surprendra pas ses fidèles lecteurs. Ses travaux antérieurs, sur l'influence du protestantisme aux débuts de la III République notamment, lui facilitaient la tâche pour la période contemporaine ; mais il n'était en rien spécialiste de la période moderne. Or, prouesse dans la prouesse, c'est celle-ci qui occupe la majeure partie du volume : un peu plus de $75 \%$ du texte contre un peu moins de $25 \%$ pour la période contemporaine, après 1789. Tout se passe donc comme si l'auteur, entraîné avec passion dans les siècles de fondation par une historiographie foisonnante, s'était pris au jeu, quitte à résumer plus sobrement ce qu'il connaissait le mieux au départ. Le résultat est saisissant : sur la violence des guerres de Religion du XVI ${ }^{\mathrm{e}}$ siècle ou sur l'Europe française du Refuge au XVIII ${ }^{\mathrm{e}}$, bien des chapitres du livre sont appelés à devenir des classiques. Mais le contemporanéiste n'est jamais très loin, qui multiplie les comparaisons avec des situations récentes connues de lui. Tellement qu'il juge utile de s'en justifier : "avoir travaillé sur les drames du $\mathrm{xx}^{\mathrm{e}}$ siècle n'est pas la plus mauvaise façon de relire ceux des guerres de Religion ou de la Révocation» (p. 10). Bien que le recours aux notions de brutalisation, de nettoyage confessionnel, d'apartheid, voire de génocide (arménien) prête à discussion, le déplacement voulu qu'ils imposent dans le temps comme dans l'espace fait souvent mouche. La comparaison entre l'exil des protestants après 1685 et des situations antérieures, l'expulsion des Juifs, puis des morisques, de la péninsule ibérique aux $\mathrm{XV}^{\mathrm{e}}$ et $\mathrm{XVI}^{\mathrm{e}}$ siècles est tout aussi éclairante (p. 754-759). Chemin faisant, Cabanel multiplie les analyses et les mises au point qui feront date : le mythique édit de Nantes de 1598 était bien plus favorable au catholicisme qu'au protestantisme qu'il circonscrivait dans des limites facilitant ensuite son étranglement; l'exil des protestants après sa révocation n'a pas entraîné le déclin économique du royaume de France; si l'édit de 1787 a permis la régularisation de leur état civil, c'est à tort qu'on le baptise édit de tolérance, car il maintient à leur détriment une intolérance de fait. Les apports de cette somme, fondée sur le dernier état de l'historiographie, paraissent inépuisables.

7 Aussi riche soit-il, l'ouvrage pâtit toutefois de choix qui ne font que prolonger ceux de ses prédécesseurs, de génération en génération. Trois principalement. Histoire des protestants en France ? S'il s'agit de la France dans ses frontières actuelles, le compte n'y est pas. Les protestants à majorité luthérienne d'Alsace (dans le royaume de France 
depuis 1648) ou du pays de Montbéliard (français depuis 1801), n'apparaissent vraiment qu'avec le paternalisme de leurs industriels au XIX ${ }^{\mathrm{e}}$ siècle. Si bien des traits les unissent à leurs coreligionnaires d'Allemagne, et si les Alsaciens bénéficient d'un régime des cultes différent, ils n'en sont pas moins, eux aussi, des " protestants en France ». On rêve d'une histoire globale de ceux-ci qui les réintègrerait dans l'épure, plutôt que de les cantonner dans une historiographie spécifique. Les Alsaciens ont-ils pâti de la Révocation de 1685 ? Que mettre sous l'étiquette piétisme? N'y a-t-il de luthériens qu'aux marges de l'Hexagone? Quid des luthériens parisiens et de leur tendance " haute Église » depuis le $\mathrm{XIX}^{\mathrm{e}}$ siècle ? La remarque vaut aussi pour les baptistes et autres non concordataires du XIX e siècle, tellement réduits à la portion congrue (p. 959-961) qu'on éprouve des difficultés à comprendre leur irruption sur la scène protestante au xxI (l'un d'entre eux, Claude Baty ${ }^{1}$, n'était-il pas, jusqu'en 2013, le président de la Fédération protestante de France ?). Bref, l'histoire que déroule Patrick Cabanel est moins celle des protestants que des huguenots, c'est-à-dire des héritiers français de Calvin dans la « France de l'intérieur ».

Un tel choix explique qu'elle soit aussi une histoire de leur répression, dominée par la violence, la guerre, l'emprisonnement ou l'exil, si prégnants dans la mémoire huguenote que partage l'auteur dont la maison porte encore la trace du grand «brûlement » des Cévennes de 1703 (p. 703-704). Un comptage sommaire montre qu'un minimum de trois cent vingt-cinq pages est consacré à l'histoire de cette victimisation. L'auteur pourra rétorquer à bon droit qu'il ne fait que restituer l'existence quotidienne des huguenots entre 1550 et 1787. D'autres choix de détail n'en sont pas moins instructifs : si le tableau de la structuration de la Religion Prétendue Réformée sous le régime de l'édit de Nantes est copieux et complet (p. 392-506), celui de ses répliques au XVIII ${ }^{e}$ siècle (p. 868-880), et même au XIXe (l'évocation de la "Terreur blanche» de 1815, dernier regain de persécution, a droit à sept pages, 1008-1015, alors la normalisation qui la suit n'en a que trente-deux, Réveil compris, 945-978) sont assez brefs, comme si les tribulations des huguenots en France et dans le monde intéressaient plus Patrick Cabanel que leurs (rares) périodes de tranquillité. D'une certaine manière, malgré toutes les garanties scientifiques dont il s'entoure, ne peut-on lire son livre, comme le prolongement historien d'une tradition qui va de l'Histoire des martyrs de Crespin à celle des pasteurs du Désert de Napoléon Peyrat, en passant par les Tragiques d'Agrippa d'Aubigné et l'Accomplissement des prophéties de Jurieu? L'histoire d'une religion-mémoire de la persécution pour laquelle Patrick Cabanel a forgé avec bonheur le terme de "huguenotisme" à partir de ses entreprises mémorielles du XIX ${ }^{e}$ siècle. Or plus le protestantisme se diversifie en France du XIX ${ }^{e}$ au XXI ${ }^{e}$ siècle et moins ce huguenotisme peut prétendre en assumer la pluralité.

Troisième choix et troisième limite, exposés d'emblée : «ce livre est donc l'histoire non pas d'une théologie, ni même d'une Église, mais d'une minorité d'hommes et de femmes ». Elle se veut même "une autre histoire de la France» (p.11), plurielle, et non plus seulement catholique ou laïque, voire "catholaïque ». L'ambition est réalisée : Patrick Cabanel fournit une remarquable histoire démographique, sociale, culturelle et politique de la minorité réformée de "France de l'intérieur ", puisée dans une historiographie qui dépasse de beaucoup l'histoire dite religieuse : après l'avoir lu on sait tout, ou presque tout, sur la rétraction géographique de la RPR depuis le milieu du XVI ${ }^{e}$ siècle, sur son ascension financière et sociale au Refuge et au XIX ${ }^{e}$ siècle ou sur sa propension au vote à gauche jusqu'aux dernières décennies. Mais qu'est-ce qui fait l'unité profonde de cette minorité, de ces hommes et de ces femmes, sinon leur adhésion à une manière spécifique d'envisager et de vivre le christianisme, sur laquelle ce gros livre ne s'appesantit guère. 
On comprend que son auteur ait voulu sortir d'une histoire ecclésiastique recroquevillée sur les décisions des synodes ou sur des débats théologiques étranges aux yeux du profane. Les querelles entre arminiens et gomaristes français au XVII ${ }^{\mathrm{e}}$ siècle ou entre libéraux et orthodoxes au XIX ${ }^{\mathrm{e}}$ sont ainsi réduites à la portion congrue. Mais là n'est pas l'essentiel. Quelles étaient la foi et la vie religieuse de tous ces gens disposés au martyr ou aux galères plutôt que de les abjurer ? Il y a de très bonnes pages sur leur enracinement dans ce que les chrétiens appellent l'Ancien Testament et sur le " patois de Canaan » qui en résulte. Mais au-delà ? La confession de foi de La Rochelle de 1559, qui a fait autorité chez les réformés jusqu'en 1872, est dite de belle « frappe calvinienne » (p. 434), sans que son contenu soit explicité. Les notations éparses sur la vie religieuse des populations réformées auraient mérité d'être regroupées, lors des phases d'accalmie, pour faire droit au meilleur du renouvellement récent de l'histoire religieuse. Que croyaient-ils et comment cette croyance se manifestait-elle quotidiennement? Exemple: une certaine réserve se manifeste à l'encontre de l'empreinte des Lumières sur la foi des reconstructeurs du XVIII ${ }^{\mathrm{e}}$ siècle et de leurs émules $d u \mathrm{XIX}^{\mathrm{e}}$, contre lesquels naît le Réveil. Mais elle n'est pas documentée : en quoi le théisme ou le rationalisme ambiant ont-ils alors contaminé la foi réformée ? And so on... Beaucoup de questions posées par l'existence chrétienne (ô Karl Barth!) des huguenots, pasteurs, cadres laïques et simples fidèles demeurent sans réponse dans un livre par ailleurs exhaustif sur leur existence profane ou sur leurs tribulations, leurs souffrances et leur mort. On le regrette d'autant plus que «le Cabanel » ne sera pas remplacé de sitôt !

\section{NOTES}

1. Qui est membre des Églises évangéliques libres, et non des Églises réformées évangéliques indépendantes, p. 1184.

\section{AUTEUR}

\section{ÉTIENNE FOUILLOUX}

Laboratoire historique Rhône-Alpes (Larhra), etienne.fouilloux@wanadoo.fr 